\title{
Finding stability regions for preserving efficiency classification of variable returns to scale technology in data envelopment analysis
}

\author{
P. Zamani ${ }^{1} \cdot$ M. Borzouei ${ }^{2}$
}

Received: 5 September 2014 / Accepted: 7 June 2016/Published online: 21 June 2016

(C) The Author(s) 2016. This article is published with open access at Springerlink.com

\begin{abstract}
This paper addresses issue of sensitivity of efficiency classification of variable returns to scale (VRS) technology for enhancing the credibility of data envelopment analysis (DEA) results in practical applications when an additional decision making unit (DMU) needs to be added to the set being considered. It also develops a structured approach to assisting practitioners in making an appropriate selection of variation range for inputs and outputs of additional DMU so that this DMU be efficient and the efficiency classification of VRS technology remains unchanged. This stability region is simply specified by the concept of defining hyperplanes of production possibility set of VRS technology and the corresponding halfspaces. Furthermore, this study determines a stability region for the additional DMU within which, in addition to efficiency classification, the efficiency score of a specific inefficient DMU is preserved and also using a simulation method, a region in which some specific efficient DMUs become inefficient is provided.
\end{abstract}

Keywords Data envelopment analysis · Efficiency · Variable returns to scale technology $\cdot$ Stability region . Defining hyperplane

\section{P. Zamani}

zamany2007@yahoo.com

1 Faculty of Engineering, Qom Branch, Islamic Azad University, Qom, Iran

2 Department of Mathematics, Science and Research Branch, Islamic Azad University, Tehran, Iran

\section{Introduction}

Data envelopment analysis (DEA) is a mathematical programming technique to evaluate relative efficiency of decision making units (DMUs) with multiple input-output. An important feature of this DEA technique which has been studied by many researchers is sensitivity analysis. During the recent years, the issue of sensitivity and stability of DEA results has been extensively studied (see for example Jahanshahloo et al. 2004, 2005a, b). The topic of sensitivity (=stability or robustness) analysis has taken a variety of forms in the DEA literature. One type of DEA sensitivity analysis is based upon data variations. The first DEA sensitivity analysis paper by Charnes et al. (1985) examined change in a single output. This is followed by a series of sensitivity analysis articles by Charnes and Neralic $(1989 a, b)$ in which sufficient conditions preserving efficiency for data variations of the test efficient DMU are determined. In other words, only subset of stability region has been achieved by their method. Moreover, the existence of alternative optimal bases is another defect of this method. Another type of DEA sensitivity analysis is based on super-efficiency DEA approach in which a test DMU is not included in reference set (see for example Andersen and Petersen 1993; Seiford and Zhu 1999: Charnes et al. 1992). In these methods, the variation range for inputs and outputs of DMUs has been attained so that the efficiency classification is preserved or an inefficient unit becomes efficient or vice versa. In this field, Zhu (1996) and Seiford and Zhu (1998) obtained the necessary and sufficient condition for preserving efficiency of a test DMU. In fact, the entire (largest) stability region which encompasses that of Charnes et al. (1992) has been obtained. Afterwards, Zhu (2001) used the super-efficiency approach in DEA sensitivity analysis for preserving a DMU's efficiency 
classification when various data changes are applied to all DMUs. In this method, variable percentage data changes are assumed for a test DMU and for the remaining DMUs. Also, he considered the worst-case analysis where the efficiency of the test DMU is deteriorating while the efficiencies of the other DMUs are improving. Cooper et al. (2001) have studied another research for the sensitivity of DEA results to variations in the data. The newer method makes it possible to determine ranges within which all data may be varied for any DMU before a reclassification from efficient to inefficient status (or vice versa) occurs. The DEA sensitivity analysis methods that authors have just reviewed are all confined to stability of DEA results under data variations for inputs and outputs of DMUs. Other topics such as sensitivity analysis to model changes or diminution or augmentation of the number of DMUs are not examined in detail. Therefore, the purpose of this article is to propose such new type of DEA sensitivity analysis. From this viewpoint, the suggested method, in comparison with previous methods, provides a more comprehensive framework for sensitivity analysis in DEA.

In addition to DEA, sensitivity analysis is used in many fields, including industry. Pitchipoo et al. (2013) proposed an appropriate platform for process industries in selecting suppliers. In their research, the sensitivity analysis was performed to improve the robustness of the results with regard to the relative importance of the evaluation criteria and the parameters of the evaluation process. Singh et al. (2012) have studied the steady state behavior of a single server queueing model with vacation and varying arrival rates. They also used the sensitivity analysis to explore the effect of different parameters on the performance measures. Faghihinia and Mollaverdi (2012) presented a multi-criterion decision-making model for preventive maintenance planning. They also used sensitivity analysis to verify the robustness of certain parameters of their proposed model. Lachhwani and Poonia (2012) showed a procedure for solving multilevel fractional programming problem based on the fuzzy set theory and goal programming approach. They also provided sensitivity analysis with variation of tolerance values on decision vectors to show how the solution is sensitive to the change of tolerance values. In all of mentioned researches, Sensitivity analysis is a technique used to determine how different values of an independent variable will impact a particular dependent variable under a given set of assumptions. It is a method to predict the outcome of a decision if a situation turns out to be different compared to the key predictions. In fact, the validity proposed models is tested by the sensitivity analysis and applying it helps to understand how accurate the problem performance is (for more analysis, see also Tabrizi and Razmi 2013; Fardis et al. 2013). In a similar way, this paper is designed to examine the sensitivity of efficiency classification of variable returns to scale (VRS) technology for enhancing the credibility of DEA results in practical applications where researchers are faced with a situation in which an additional DMU needs to be added to the set of the observed DMUs, a new production possibility set (PPS) is obtained. As a result of the proposed method, the variation range for inputs and outputs of additional DMU is determined so that the additional DMU becomes efficient and the classification of efficient and inefficient DMUs of VRS technology remains unchanged; furthermore, the other special conditions are preserved. For example, the efficiency score of a specific inefficient DMU is preserved or a specific efficient DMU becomes inefficient or all DMUs on a specific defining hyperplane become inefficient.

The current article proceeds as follows: Sect. "Background" briefly reviews a mathematical basis used for this study. Also, stability radius of DEA models in various papers is defined. In Sect. "Proposed method", the proposed method is presented. Four proposed methods are applied for two numerical examples in Sect. "Numerical examples". Finally, concluding remarks are summarized in the last section.

\section{Background}

DEA is a mathematical programming technique to evaluate relative efficiency of DMUs with multiple input-output. It was introduced by Charnes et al. (1978). In their original DEA model, Charnes et al. (CCR model) proposed that the efficiency of a DMU can be obtained as the maximum of a ratio of weighted outputs to weighted inputs, subject to the condition that the same ratio for all DMUs must be less than or equal to one. The envelopment in CCR is constant returns to scale (CRS) meaning that a proportional increase (decrease) in inputs results in a proportionate increase (decrease) in outputs. However, in efficiency analysis, VRS can also be considered. Banker et al. (1984) developed the BCC model with VRS to estimate the efficiency of DMUs, which we are interested in this paper.

Suppose that we have n DMUs, where each $\mathrm{DMU}_{j}$, $j=1, \ldots, n$, produces the same s outputs in (possibly) different amounts $y_{r j}, r=1, \ldots, s$, using the same m inputs $x_{i j}$, $i=1, \ldots, m$, also in (possibly) different amounts. All data are assumed to be nonnegative, but at least one of the components of every input and output vector is positive. A pair of such a semipositive input and output vector is called an activity. The PPS is defined as the set of feasible activities. Since, we are interested in BCC models in this paper, we represent the PPS of VRS technology in the following manner: 


$$
\begin{aligned}
T_{v}= & \left\{(x, y) \mid x \geq \sum_{j=1}^{n} \lambda_{j} x_{j}, \quad y \leq \sum_{j=1}^{n} \lambda_{j} y_{j},\right. \\
& \left.\times \sum_{j=1}^{n} \lambda_{j}=1, \quad \lambda_{j} \geq 0, \quad j=1, \ldots, n\right\}
\end{aligned}
$$

Definition $1 \mathrm{DMU}_{p}$ is called to be efficient in $T_{v}$ if and only if there does not exist another $(x, y) \in T_{v}$ such that $(-x, y) \geq\left(-x_{p}, y_{p}\right)$ and $(-x, y) \neq\left(-x_{p}, y_{p}\right)$, otherwise, $\mathrm{DMU}_{p}$ is called inefficient. In other words, $\mathrm{DMU}_{p}$ is called efficient if and only if $\mathrm{DMU}_{p}$ is a non-dominated DMU in $T_{v}$.

Based on the PPS of VRS technology the envelopment form of BCC model is in the following manner:

Min $\theta$

$$
\begin{array}{ll}
\text { s.t. } & \sum_{j=1}^{n} \lambda_{j} x_{j} \leq \theta x_{p} \\
& \sum_{j=1}^{n} \lambda_{j} y_{j} \geq y_{p} \\
& \sum_{j=1}^{n} \lambda_{j}=1 \\
& \lambda_{j} \geq 0, \quad j=1, \ldots, n
\end{array}
$$

For $\mathrm{DMU}_{\mathrm{p}}$, its reference set, $E_{p}$, is defined by $\begin{aligned} E_{p} & =j\left\{\mid \lambda_{j}^{*}>0 \text { in some optimal solution of }(1)\right\} \\ & \subseteq\{1, \ldots, n\}\end{aligned}$

References of a $\mathrm{DMU}_{\mathrm{p}}$ are efficient DMUs that there is a combination of them that dominates $\mathrm{DMU}_{p}$.

Model (1) used for evaluating relative efficiency of $\mathrm{DMU}_{p}(p=1, \ldots, \mathrm{n})$ classifies them into three different categories, which are mutually disjoint.

1. $\mathrm{DMU}_{\mathrm{p}}$ is inefficient, then it is not a reference DMU and there are other DMUs in its reference set, $E_{p}$.

2. $\mathrm{DMU}_{\mathrm{p}}$ is extreme efficient, then its only reference of itself.

3. $\mathrm{DMU}_{\mathrm{p}}$ is nonextreme efficient, then in addition of itself, there is at least one other DMU in $E_{p}$.

The multiplier form of BCC model based on the dual of model (1) is as follows:

$$
\begin{array}{ll}
\operatorname{Max} & u^{t} y_{p}+u_{0} \\
\text { s.t. } & v^{t} x_{p}=1 \\
& u^{t} y_{j}-v^{t} x_{j}+u_{0} \leq 0, \quad j=1, \ldots, n \\
& u \geq 0, \quad v \geq 0, \quad u_{0} \text { free }
\end{array}
$$

where $u^{t}=\left(u_{1}, \ldots, u_{s}\right)$ and $v^{t}=\left(v_{1}, \ldots, v_{m}\right)$ are s-vector and $\mathrm{m}$-vector, respectively.

Definition 2 In (2), $\mathrm{DMU}_{\mathrm{p}}$ is efficient if and only if there exists at least one optimal solution $\left(u^{*}, v^{*}, u_{0}^{*}\right)$ for $(2)$, with $\left(u^{*}, v^{*}\right)>0$ such that $u^{*} y_{p}+u_{0}^{*}=1$; otherwise $\mathrm{DMU}_{\mathrm{p}}$ is inefficient.

In the evaluation of $\mathrm{DMU}_{\mathrm{p}}(p \in\{1, \ldots, n\})$, if $\left(u^{*}, v^{*}, u_{0}^{*}\right)$ is an optimal solution of (2), $u^{* t} y-v^{* t} x+u_{0}^{*}=0$ will be a supporting hyperplane on the PPS (Cooper et al. 1999). Consider $\mathrm{DMU}_{\mathrm{p}}$ in Fig. 1. Using model (2), it can be seen that there are alternative optimal solutions (see cooper et al. 2007) which define an infinite number of hyperplanes passing through $\mathrm{DMU}_{p}$, of which only two hyperplanes $\left(H_{1}\right.$ and $\mathrm{H}_{2}$ ) are defining hyperplanes. (for more details of defining hyperplanes and properties see Jahanshahloo et al. 2007).

The efficiency score obtained by standard DEA models cannot be used for ranking efficient DMUs. So Andersen and Petersen (1993) developed a procedure for ranking efficient units. They omitted the evaluating $\mathrm{DMU}_{p}$ from the observed DMUs. Based on the VRS technology, the new PPS is as follows:

$T_{v}^{\prime}=\left\{(x, y) \mid x \geq \sum_{\substack{j=1 \\ j \neq p}}^{n} \lambda_{j} x_{j}, y \leq \sum_{\substack{j=1 \\ j \neq p}}^{n} \lambda_{j} y_{j}, \sum_{\substack{j=1 \\ j \neq p}}^{n} \lambda_{j}=1, \lambda_{j} \geq 0, j=1, \ldots, n, j \neq p\right\}$

Let $\mathrm{DMU}_{\mathrm{p}}$ is evaluated. The AP model for ranking DMUs is as follow:

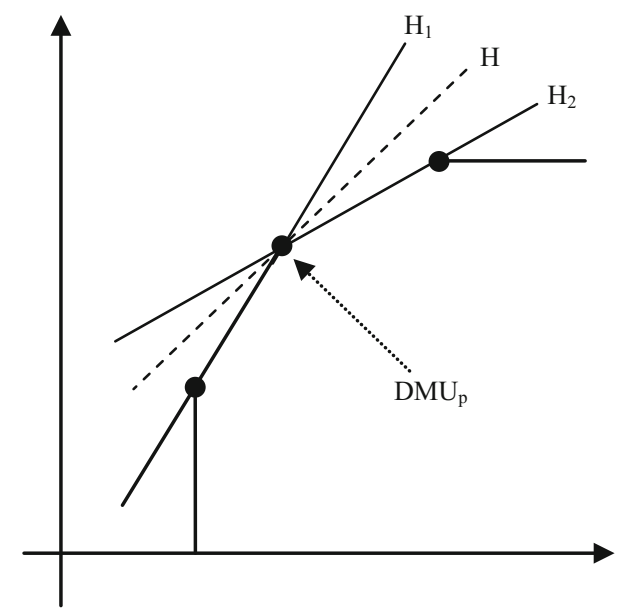

Fig. $1 H_{1}$ and $H_{2}$ are defining. $H$ is not defining 


$$
\begin{aligned}
& z_{p}= \operatorname{Min} \theta \\
& \text { s.t. } \sum_{\substack{j=1 \\
j \neq p}}^{n} \lambda_{j} x_{j} \leq \theta x_{p} \\
& \sum_{\substack{j=1 \\
j \neq p}}^{n} \lambda_{j} y_{j} \geq y_{p} \\
& \sum_{\substack{j=1 \\
j \neq p}}^{n} \lambda_{j}=1 \\
& \lambda_{j} \geq 0, \quad j=1, \ldots, n, \quad j \neq p
\end{aligned}
$$

For the optimal solution $z_{p}^{*}$ :

- If $z_{p}^{*}<1$, then $\mathrm{DMU}_{\mathrm{p}}$ is inefficient.

- If $z_{p}^{*}=1$, then $\mathrm{DMU}_{\mathrm{p}}$ is non-extreme efficient.

- If $z_{p}^{*}>1$ or (3) is not feasible, then $\mathrm{DMU}_{p}$ is extreme efficient.

Having identified efficient and inefficient DMUs in a DEA analysis, one may want to know how sensitive these identifications are to possible variations in the data. A new avenue for sensitivity analysis was opened by Charnes et al. (1992, 1996). They determined "radii of stability" within which data variations will not alter a DMU's classification from efficient to inefficient status (or vice versa). Then, Cooper et al. (2001) modified the models in Charnes et al. (1996) for sensitivity analysis of inefficient DMUs. Their model have yielded a radius of stability within which all inputs and outputs for an inefficient $\mathrm{DMU}_{\mathrm{p}}$ can be improved without producing a change from inefficient to efficient status. This means that no reclassification to efficient status will occur within the symmetric region defined by them. However, for sensitivity analysis of efficient DMUs, they used the proposed model by Charnes et al. (1996). Jahanshahloo et al. (2005b) presented a modified version of the proposed models in Cooper et al. (2001) to determine stability radius for inputs and outputs of efficient and inefficient DMUs by one-model approach. In the method proposed by Cooper et al. (2001), separate models are solved for sensitivity analysis of efficient and inefficient DMUs. Therefore, by solving $\mathrm{n}$ problems, classification of DMUs is first identified. Then, for the purpose of obtaining the variation ranges and radius of stability, at least $n$ problems are resolved. But in the latter method by solving only $n$ problems, classification, variations ranges of inputs and outputs and radius of stability of all DMUs are identified. As mentioned, stability radius is obtained from various methods to preserve DEA results under data variations of DMUs. Other topics such as sensitivity (or stability) analysis in the presence of the additional DMU are not examined yet. Therefore, the purpose of this article is to propose such new type of DEA sensitivity analysis that examined in the next section. As a result, the procedure yields an exact stability radius for the additional DMU within which the efficiency classification of VRS technology remains unchanged.

\section{Proposed method}

This section outlines a sensitivity analysis approach for DEA studies where researchers are faced with a situation in which an additional DMU needs to be added to the set being considered. As a result of proposed method, the variation range for inputs and outputs of additional DMU is determined, so that the additional DMU becomes efficient and the classification of efficient and inefficient DMUs of $T_{v}$ remains unchanged. In this paper, this region is called stability region. This stability region is specified by the concept of defining hyperplanes of PPS of VRS technology and the corresponding halfspaces.

Recall that we have $\mathrm{n}$ DMUs. By adding an additional DMU (DMU $\left.{ }_{n+1}\right)$ to the set of observed DMUs and solving the problem (3) for it, we have:

- If $z_{n+1}^{*} \leq 1$, the efficient frontier will remain unchanged.

- If $z_{n+1}^{*}>1$, the additional DMU will change the efficient frontier and some efficient DMUs may become inefficient. Hence, a method for finding a stability region in this case is presented. (It should be noted that the new efficient frontier includes the additional DMU).

Regardless of the additional DMU, we first find all efficient DMUs (with model (2)) and defining hyperplanes of $T_{v}$ (for a review see Jahanshahloo et al. 2005c, 2007, 2009) which we are interested in. It has been assumed that the redundant hyperplanes, which have no effect on the PPS, are omitted.

Let $H_{i}=\left\{(x, y) \mid u_{i}^{* t} y-v_{i}^{* t} x+u_{0 i}^{*}=0\right\}$ be one of these defining hyperplanes. Corresponding to hyperplane $H_{i}$, the halfspace $H_{i}^{+}$is defined as follows:

$H_{i}^{+}=\left\{(x, y) \mid u_{i}^{* t} y-v_{i}^{* t} x+u_{0 i}^{*} \geq 0\right\}$

After determining of $H_{i}$, we find all efficient DMUs of $T_{v}$ that lie on $H_{i}$. Let $\Omega=\left\{\mathrm{DMU}_{1}, \ldots, \mathrm{DMU}_{h}\right\}$ be the set of these DMUs. Now, we find all defining hyperplanes of $T_{v}$ (excluding $H_{i}$ ) passing from each $\operatorname{DMU}_{1}(1=1, \ldots, h)$ defined as follows:

$H_{i_{k}}=\left\{(x, y) \mid u_{i_{k}}^{* t} y-v_{i_{k}}^{* t} x+u_{0 i_{k}}^{*}=0\right\}, \quad k=1, \ldots, f$

Corresponding to hyperplane $H_{i_{k}}$, the halfspace $H_{i_{k}}^{-}$is defined as follows:

$H_{i_{k}}^{-}=\left\{(x, y) \mid u_{i_{k}}^{* t} y-v_{i_{k}}^{* t} x+u_{0 i_{k}}^{*} \leq 0\right\}, \quad k=1, \ldots, f$ 
Now, corresponding to the $i$ th defining hyperplane, we set

$S_{i}^{\prime}=\bigcap_{k=1}^{f} H_{i_{k}}^{-}, S_{i}=S_{i}^{\prime} \bigcap H_{i}^{+}, \mathrm{S}=\bigcup_{\mathrm{i}} \mathrm{S}_{\mathrm{i}}$

Theorem $1 \quad S=\bigcup_{i} S_{i}$ is a stability region for the additional DMU.

Proof Without loss of generality, we prove that the efficiency classification remains unchanged by adding an additional DMU to $S_{i}$. By contradiction, suppose that one of the efficient DMUs, say $\mathrm{DMU}_{1}$, becomes inefficient. Therefore, there is another (virtual) DMU of new PPS such that $\left(-\sum_{j=1}^{n+1} \lambda_{j} x_{j}, \sum_{j=1}^{n+1} \lambda_{j} y_{j}\right) \geq\left(-x_{l}, y_{l}\right)$ and $\left(-\sum_{j=1}^{n+1} \lambda_{j} x_{j}\right.$, $\left.\sum_{j=1}^{n+1} \lambda_{j} y_{j}\right) \neq\left(-x_{l}, y_{l}\right)$.

Suppose that $u_{i_{k}}^{* t} y_{l}-v_{i_{k}}^{* t} x_{l}+u_{0 i_{k}}^{*}=0$ is the passing defining hyperplane (excluding $H_{i}$ ) from $\mathrm{DMU}_{1}$. Hence

$u_{i_{k}}^{* t} \sum_{j=1}^{n+1} \lambda_{j} y_{j}-v_{i_{k}}^{* t} \sum_{j=1}^{n+1} \lambda_{j} x_{j}>u_{i_{k}}^{* t} y_{l}-v_{i_{k}}^{* t} x_{l}$

Set $u_{i_{k}}^{* t} y_{l}-v_{i_{k}}^{* t} x_{l}=-u_{0 i_{k}}^{*}$. Then substitute it in the above expression to obtain

$u_{i_{k}}^{* t} \sum_{j=1}^{n+1} \lambda_{j} y_{j}-v_{i_{k}}^{* t} \sum_{j=1}^{n+1} \lambda_{j} x_{j}+u_{0 i_{k}}^{*}>0$

This implies that $\left(\sum_{j=1}^{n+1} \lambda_{j} x_{j}, \sum_{j=1}^{n+1} \lambda_{j} y_{j}\right) \notin S_{i}$. Consequently there is no another (virtual) DMU of new PPS which dominates $\mathrm{DMU}_{1}$. Thus, $\mathrm{DMU}_{1}$ remains efficient.

Theorem 2 If the additional DMU does not belong to the reference set of a specific inefficient $D M U_{p}$ the efficiency score of $D M U_{p}$ will remain unchanged.

Proof Since additional DMU does not belong to the reference set of $\mathrm{DMU}_{p}, \lambda_{n+1}^{*}=0$ (for each optimal solutions of (1)), and elimination of $\mathrm{DMU}_{n+1}$ does not change the optimal objective function value of (1). Therefore, the efficiency score of $\mathrm{DMU}_{p}$ remains unchanged.

Now, we obtain a stability region for the additional DMU within which, in addition to efficiency classification, the efficiency score of a specific inefficient DMU is preserved. In other words, by adding the additional DMU, the status of a special inefficient DMU does not change (does not become worse). One of the practical applications of this subject is when an additional DMU needs to be added to the set being considered, but by adding this additional DMU, Decision Maker (DM) does not want to deteriorate the situation of the other inefficient unit that is in the sensitive position.

For this purpose, we first find all efficient DMUs and defining hyperplanes of $T_{v}$. Then by omission of defining hyperplane that includes the reference set of $\mathrm{DMU}_{p}$, we repeat previous methodology for the remaining defining hyperplanes of $T_{v}$.

Sometimes, in practice, DM faced problems in which he wants to construct a new unit or department so that the competitor efficient units become inefficient and the efficiency classification of the other units remains unchanged. In this way, we now determine a stability region for additional DMU that an efficient $\mathrm{DMU}_{p}$ becomes inefficient and also the efficiency classification of the other DMUs remains unchanged.

Again we first find all efficient DMUs and defining hyperplanes of $T_{v}$ that passes through $\mathrm{DMU}_{p}$. Corresponding to these hyperplanes, the halfspaces $H_{i}^{+}$ $(i=1, \ldots, 1)$ are defined in the following manner:

$H_{i}^{+}=\left\{(x, y) \mid u_{i}^{* t} y-v_{i}^{* t} x+u_{0 i}^{*}>0\right\}, \quad i=1, \ldots, l$

Suppose that $\Omega=\left\{\mathrm{DMU}_{1}, \ldots, \mathrm{DMU}_{h}\right\}$ be the set of efficient DMUs of $T_{v}$ that lies on $H_{1}$ or $H_{2}$ or .... or $H_{l}$. So, we find all defining hyperplanes of $T_{v}$ (excluding $H_{i}$ ) that $\mathrm{DMU}_{1}, \ldots, \mathrm{DMU}_{h}$ lie on them. Let

$$
H_{i_{k}}^{-}=\left\{(x, y) \mid u_{i_{k}}^{* t} y-v_{i_{k}}^{* t} x+u_{0 i_{k}}^{*} \leq 0\right\}, \quad(k=1, \ldots, f)
$$

be the halfspaces obtained by these hyperplanes.

Corresponding to $\mathrm{DMU}_{\mathrm{p}}$, set

$S_{1}=\bigcap_{i=1}^{l} H_{i}^{+}, \quad S_{2}=\bigcap_{k=1}^{f} H_{i_{k}}^{-}, \quad S=S_{1} \bigcap S_{2}$

Theorem $3 \quad S=S_{1} \bigcap S_{2}$ is a stability region for the additional DMU that an efficient $D M U_{p}$ becomes inefficient and also the efficiency classification of the other DMUs remains unchanged.

Proof By contradiction, suppose that $\mathrm{DMU}_{\mathrm{p}}$ remains efficient in the presence of additional DMU. Hence, there is at least a defining hyperplane of new PPS, say $H_{p}$, which passes through $\mathrm{DMU}_{p}$. Since $\mathrm{DMU}_{p}$ is efficient, there is no point of efficient facet contained in this hyperplane dominated by (virtual) DMUs that belong to new PPS. Consequently, $\mathrm{DMU}_{n+1}$ belongs to the halfspace $H_{p}^{-}$of passing defining hyperplane from $\mathrm{DMU}_{p}$. So, $\mathrm{DMU}_{n+1} \notin S$ is a contradiction.

In the following, we wish to determine a stability region for the additional DMU in which all DMUs that lie on a specific defining hyperplane (H) of $T_{v}$ become inefficient.

Suppose that $\Omega=\left\{\mathrm{DMU}_{1}, \ldots, \mathrm{DMU}_{h}\right\}$ be the set of efficient DMUs that lies on $H$. So, we find all defining hyperplanes of $T_{v}$ (excluding $H$ ) which $\mathrm{DMU}_{1}, \ldots, \mathrm{DMU}_{h}$ lie on them. Corresponding to these hyperplanes, the halfspaces $H_{i}^{+}(i=1, \ldots, l)$ are defined as follows:

$H_{i}^{+}=\left\{(x, y) \mid u_{i}^{* t} y-v_{i}^{* t} x+u_{0 i}^{*}>0\right\}, \quad i=1, \ldots, l$ 
Corresponding to the remaining defining hyperplanes of $T_{v}$, we find halfspaces $H_{i_{k}}^{-}(k=1, \ldots, f)$

Set

$S_{1}=\bigcap_{i=1}^{l} H_{i}^{+}, \quad S_{2}=\bigcap_{k=1}^{f} H_{i_{k}}^{-}, \quad S=S_{1} \bigcap S_{2}$

In the first glance, it seems that there should be enough information locally to determine whether a new DMU is inside a stability region without necessarily finding a full representation of this region using hyperplanes with resolving the entire DEA problem whenever there is a new exterior DMU and hence this leads to solve $(n+1)$ linear programs whereas the scheme proposed requires a complete description of $T_{v}$ in terms of hyperplanes which is computationally confusing.

There is one obvious alternative:

Despite the apparent simplicity of this approach, it has been noticed that the procedure needs to resolve $(n+1)$ linear programs in the presence of additional DMU and the problem actually grows every time a new DMU is considered. In this method we also cannot determine the variation range of inputs and outputs and stability region of additional DMU. However, in our method, since $\mathrm{n}$ earlier DMUs evaluated by multiplier form and the classification of efficient and inefficient DMUs specified, we can easily find all defining hyperplanes of $T_{v}$ with applying the proposed algorithm in Jahanshahloo et al. (2009). On the other hand, using optimal solutions of the multiplier form when $n$ DMUs are evaluated, we can determine the stability region of additional DMU which satisfies the special conditions for the rest of DMUs (For example, the efficiency score of a specific inefficient unit must be preserved or some competitor efficient units need to be inefficient in the presence of additional DMU).

\section{Numerical examples}

To illustrate the proposed method, we present two examples.

Example 1 Consider the six DMUs with single input and single output as defined in Table 1.

By evaluating these DMUs by means of the model (2), we find out that the DMUs A, $B, C$ and $D$ are efficient and DMUs $E$ and $F$ are inefficient.

Table 1 DMUs' data of Example 1

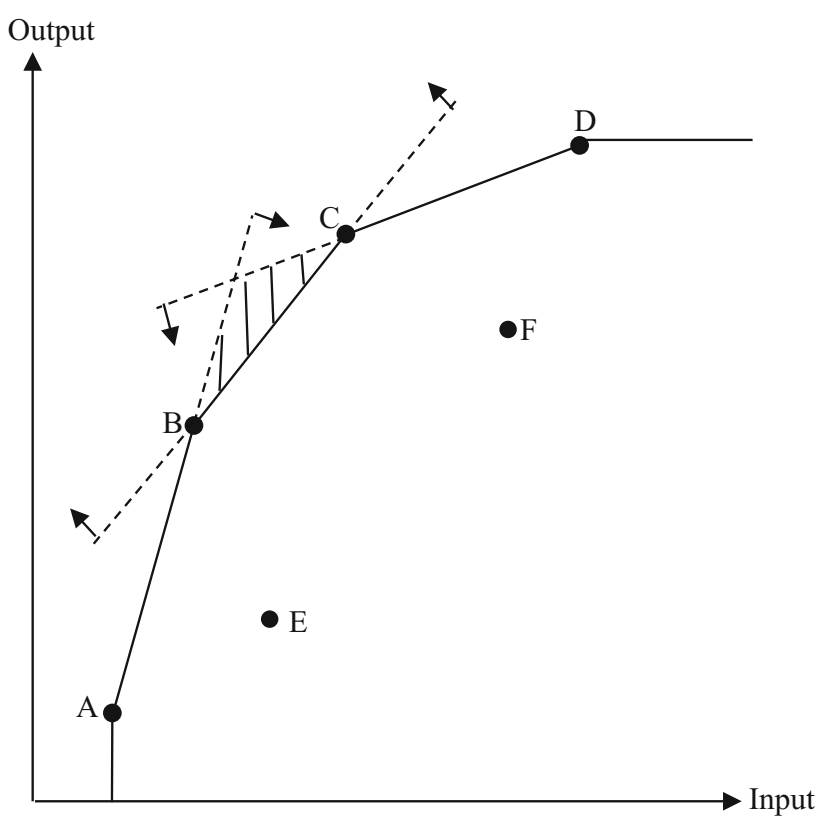

Fig. 2 Stability region for the additional DMU corresponding to $H_{B C}$

We find all defining hyperplanes of $T_{v}$ defined in the following manner:

$$
\begin{aligned}
H_{A} & =\{(x, y) \mid-x+1=0\} \\
H_{A B} & =\{(x, y) \mid y-3 x+2=0\} \\
H_{B C} & =\{(x, y) \mid y-x-2=0\} \\
H_{C D} & =\{(x, y) \mid 3 y-x-14=0\} \\
H_{D} & =\{(x, y) \mid y-7=0\}
\end{aligned}
$$

For finding a stability region for the additional DMU, we first consider $H_{B C}$. The set of all DMUs that lie on $H_{B C}$, $\Omega$, is defined by

$\Omega=\left\{\mathrm{DMU}_{B}, \mathrm{DMU}_{C}\right\}$

Hence, we will have

$$
\begin{aligned}
H_{B C}^{+} & =\{(x, y) \mid y-x-2 \geq 0\} \\
H_{A B}^{-} & =\{(x, y) \mid y-3 x+2 \leq 0\} \\
H_{C D}^{-} & =\{(x, y) \mid 3 y-x-14 \leq 0\} \\
S_{B C}^{\prime} & =H_{A B}^{-} \bigcap H_{C D}^{-} \\
& =\{(x, y) \mid y-3 x+2 \leq 0,3 y-x-14 \leq 0\} \\
S_{B C} & =S_{B C}^{\prime} \bigcap H_{B C}^{+} \\
& =\{(x, y) \mid y-3 x+2 \leq 0, \quad 3 y-x-14 \leq 0, \quad y-x-2 \geq 0\}
\end{aligned}
$$

Figure 2 illustrates a stability region for the additional DMU corresponding to $H_{B C}$. So, we repeat the previous methodology for each defining hyperplane of $T_{v} . S=\cup_{i} S_{i}$ is a stability region of this problem.

Now, we obtain a stability region for the additional DMU within which, in addition to efficiency classification, the efficiency score of $\mathrm{DMU}_{F}$ is preserved. 


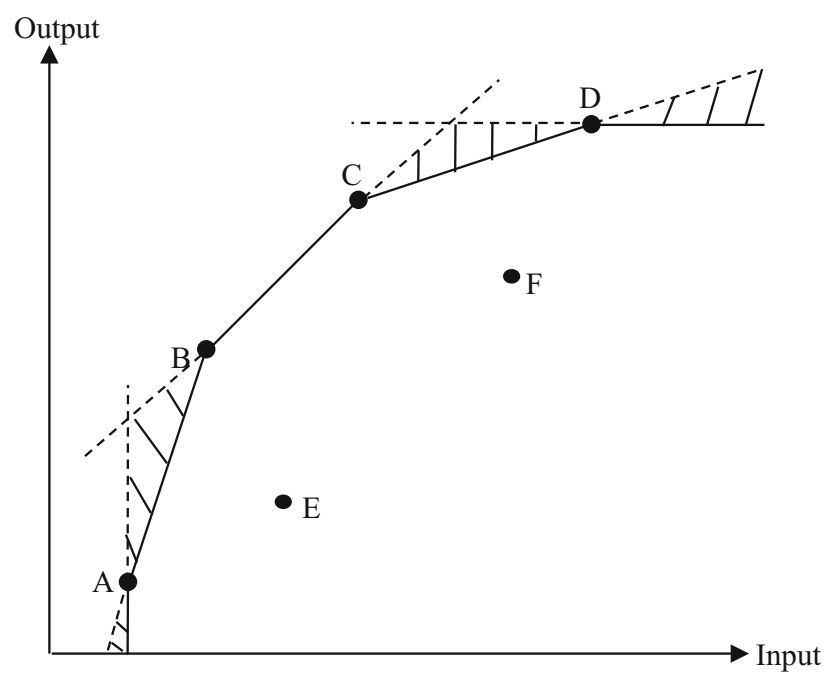

Fig. 3 Stability region for the additional DMU where the efficiency score of $\mathrm{DMU}_{F}$ is preserved

Table 2 DMUs' data of Example 2

\begin{tabular}{llllllll}
\hline DMUs & $A$ & $B$ & $P$ & $C$ & $D$ & $E$ & $F$ \\
\hline Input & 1 & 2 & 4 & 6 & 10 & 4 & 6 \\
Output & 1 & 4 & 7 & 8 & 9 & 4 & 5 \\
\hline
\end{tabular}

By omission of defining hyperplane that included the reference set of $\mathrm{DMU}_{F}, H_{B C}$, we get:

$S_{1}=\{(x, y) \mid-x+1 \geq 0, y-3 x+2 \leq 0\}$

$S_{2}=\{(x, y) \mid-y-3 x+2 \geq 0, y-x-2 \leq 0,-x+1 \leq 0\}$

$S_{3}=\{(x, y) \mid 3 y-x-14 \geq 0, y-x-2 \leq 0, y-7 \leq 0\}$

$S_{4}=\{(x, y) \mid y-7 \geq 0,3 y-x-14 \leq 0\}$

$S=\bigcap_{i=1}^{4} S_{i}$ is a stability region for the additional DMU as shown in Fig. 3.

Example 2 Consider the data setting of Table 2 contain 7 DMUs with single input and single output.

The results of model (2) show five DMUs, $A, B, P$, $C$ and $D$ are efficient.

Now, we are interested to determine a stability region for the additional DMU such that $\mathrm{DMU}_{P}$ becomes inefficient and also the efficiency classification of the other DMUs remains unchanged. We first find all defining hyperplanes of $T_{v}$ which are passing from $\mathrm{DMU}_{P}$. These hyperplanes and corresponding halfspaces $\left(H_{i}^{+}\right)$are as follows:

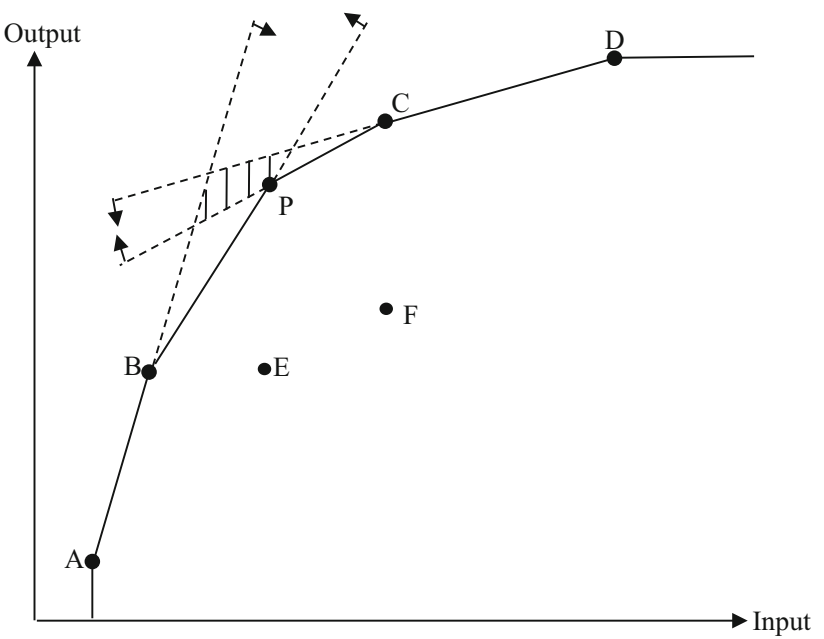

Fig. 4 Stability region for the additional DMU where $\mathrm{DMU}_{p}$ becomes inefficient

$$
\begin{aligned}
& H_{B P}=\{(x, y) \mid 2 y-3 x-2=0\} \\
& H_{P C}=\{(x, y) \mid 2 y-x-10=0\} \\
& H_{B P}^{+}=\{(x, y)|2 y-3 x-2\rangle 0\} \\
& H_{P C}^{+}=\{(x, y)|2 y-x-10\rangle 0\}
\end{aligned}
$$

$\Omega=\left\{\mathrm{DMU}_{B}, \mathrm{DMU}_{C}\right\}$ is the set of efficient DMUs of $T_{v}$ that lies on $H_{B P}$ or $H_{P C}$. The defining hyperplanes passing from the members of set $\Omega$ (excluding $H_{B P}$ and $H_{P C}$ ) and corresponding halfspaces $\left(H_{i_{k}}^{-}\right)$are as follows:

$$
\begin{aligned}
& H_{A B}=\{(x, y) \mid y-3 x+2=0\} \\
& H_{C D}=\{(x, y) \mid 4 y-x-26=0\} \\
& H_{A B}^{-}=\{(x, y) \mid y-3 x+2 \leq 0\} \\
& H_{C D}^{-}=\{(x, y) \mid 4 y-x-26 \leq 0\}
\end{aligned}
$$

Therefore, we will have:

$$
\begin{aligned}
S_{1}= & H_{B P}^{+} \bigcap H_{P C}^{+} \\
= & \{(x, y)|2 y-3 x-2\rangle 0,2 y-x-10\rangle 0\} \\
S_{2}= & H_{A B}^{-} \bigcap H_{C D}^{-} \\
= & \{(x, y) \mid y-3 x+2 \leq 0,4 y-x-26 \leq 0\} \\
S= & S_{1} \bigcap S_{2} \\
= & \{(x, y)|2 y-3 x-2\rangle 0,2 y-x-10\rangle 0, y-3 x \\
& +2 \leq 0,4 y-x-26 \leq 0\}
\end{aligned} .
$$

$S$ is a stability region as shown in Fig. 4 .

We wish to determine a stability region in which $\mathrm{DMU}_{\mathrm{P}}$ and $\mathrm{DMU}_{\mathrm{C}}$ that lie on $H_{P C}$ become inefficient as shown in Fig. 5.

For this purpose, we perform as follows: 


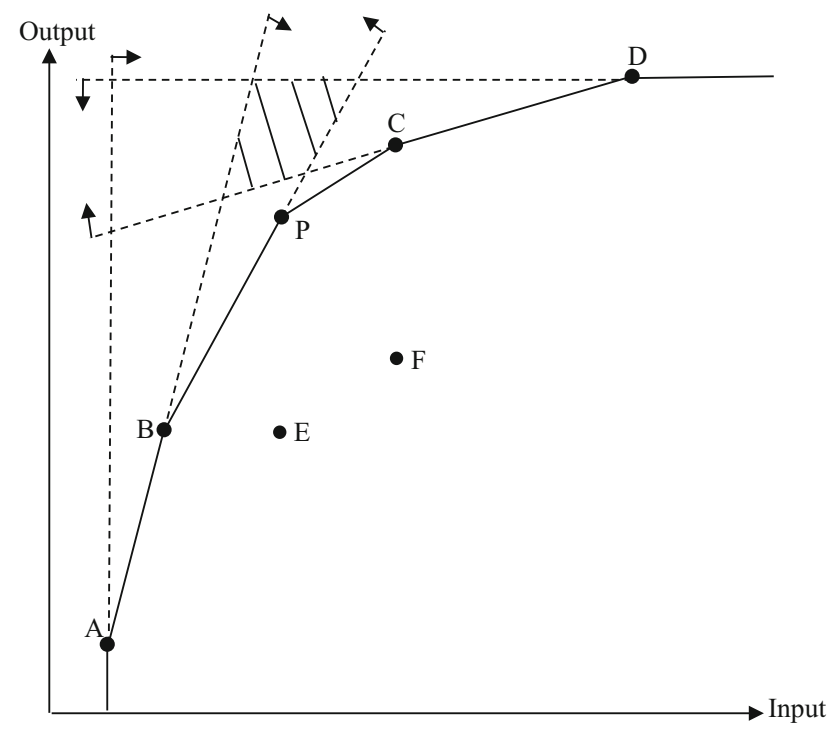

Fig. 5 Stability region for the additional DMU where all efficient DMUs that lie on $H_{P C}$ need to be inefficient

$$
\begin{aligned}
H_{B P}^{+}= & \{(x, y)|2 y-3 x-2\rangle 0\} \\
H_{C D}^{+}= & \{(x, y)|4 y-x-26\rangle 0\} \\
H_{A}^{-}= & \{(x, y) \mid-x+1 \leq 0\} \\
H_{A B}^{-}= & \{(x, y) \mid y-3 x+2 \leq 0\} \\
H_{D}^{-}= & \{(x, y) \mid y-9 \leq 0\} \\
S_{1}= & H_{B P}^{+} \bigcap H_{C D}^{+} \\
= & \{(x, y)|2 y-3 x-2\rangle 0,4 y-x-26\rangle 0\} \\
S_{2}= & H_{A}^{-} \bigcap H_{A B}^{-} \bigcap \mid H_{D}^{-} \\
= & \{(x, y) \mid-x+1 \leq 0, y-3 x+2 \leq 0, y-9 \leq 0\} \\
S= & S_{1} \bigcap S_{2} \\
= & \{(x, y)|2 y-3 x-2\rangle 0,4 y-x-26\rangle 0,-x+1 \leq 0, \\
& y-3 x+2 \leq 0, y-9 \leq 0\}
\end{aligned}
$$

\section{Conclusion}

In this paper, a new version of proposed methods for sensitivity analysis DMUs has been presented. The problem is to assess the sensitivity and stability of efficiency classification of VRS technology when an additional DMU needs to be added to the set being considered. As a result of proposed method, a stability region for the additional DMU is specified within which the additional DMU becomes efficient and the classification of efficient and inefficient DMUs of $T_{v}$ remains unchanged. This stability region is simply specified by the concept of defining hyperplanes of PPS of VRS technology and the corresponding halfspaces. Furthermore, this stability region is determined under the special conditions in which the efficiency score of a specific inefficient DMU remains unchanged or some specific efficient DMUs become inefficient. Practical applications of each method provided in this article could be the reason for the contribution of this work. Also, the following directions can be applied for future researches:

1. Finding a stability region for the additional DMU under new condition in which the ranking of efficient DMUs does not change.

2. Finding a stability region for the additional DMU under new condition in which returns to scale of the other DMUs does not change.

3. Developing the proposed methods for preserving efficiency classification of constant returns to scale (CRS) technology in data envelopment analysis.

4. Determining the largest stability region for the additional DMU under different proposed conditions in Sect. "Prosposed method".

The largest stability region means that if the additional DMU is placed outside this region, at least one of the efficient units will move interior of $T_{v}$ and becomes inefficient.

Open Access This article is distributed under the terms of the Creative Commons Attribution 4.0 International License (http://creative commons.org/licenses/by/4.0/), which permits unrestricted use, distribution, and reproduction in any medium, provided you give appropriate credit to the original author(s) and the source, provide a link to the Creative Commons license, and indicate if changes were made.

\section{References}

Andersen P, Petersen NC (1993) A procedure for ranking efficient units in data envelopment analysis. Manage Sci 39:1261-1264. doi:10.1287/mnsc.39.10.1261

Banker RD, Charnes A, Cooper WW (1984) Some models for estimating technical and scale inefficiencies in data envelopment analysis. Manage Sci 30:1078-1092. doi:10.1287/mnsc.30.9. 1078

Charnes A, Neralic L (1989a) Sensitivity analysis in data envelopment analysis 1. Glasnik Matematicki 24(44):211-226

Charnes A, Neralic L (1989b) Sensitivity analysis in data envelopment analysis 2. Glasnik Matematicki 24(44):449-463

Charnes A, Cooper WW, Rhodes E (1978) Measuring the efficiency of decision making units. Eur J Oper Res 2:429-444. doi:10. 1016/0377-2217(78)90138-8

Charnes A, Cooper WW, Lewin AY, Morey RC, Rousseau JJ (1985) Sensitivity and stability analysis in DEA. Ann Oper Res 2:139-156. doi:10.1007/BF01874736

Charnes A, Haag S, Jaska P, Semple J (1992) Sensitivity of efficiency calculations in the additive model of data envelopment analysis. Int J Syst Sci 23:789-798

Charnes A, Rousseau JJ, Semple JH (1996) Sensitivity and stability of efficiency classifications in DEA. J Prod Anal 7:5-18. doi:10. 1007/BF00158473

Cooper WW, Seiford LM, Tone K (1999) Data envelopment analysis: A comprehensive text with models, Application, References and DEA-Solver software. Kluwer Academic publishers, Berlin 
Cooper WW, Li S, Seiford LM, Tone K, Thrall RM, Zhu J (2001) Sensitivity and stability analysis in DEA: some recent development. J Prod Anal 15(3):217-246. doi:10.1023/A:1011128409257

Cooper WW, Ruiz JL, Sirvent I (2007) Choosing weights from alternative optimal solutions of dual multiplier models in DEA. Eur J Oper Res 180:443-458. doi:10.1016/j.ejor.2006.02.037

Faghihinia E, Mollaverdi N (2012) Building a maintenance policy through a multi-criterion decision-making model. J Ind Eng Int 8(14):1-15. doi:10.1186/2251-712x-8-14

Fardis F, Zandi A, Ghezavati V (2013) Stochastic extension of cellular manufacturing systems: a queuing-based analysis. J Ind Eng Int 9(20):1-8. doi:10.1186/2251-712x-9-20

Jahanshahloo GR, Hosseinzadeh Lotfi F, Moradi M (2004) Sensitivity and stability analysis in DEA with interval data. Appl Math Comput 156:463-477. doi:10.1016/j.amc.2003.08.005

Jahanshahloo GR, Hosseinzadeh Lotfi F, Shoja N, Sanei M, Tohidi G (2005a) Sensitivity and stability analysis in DEA. Appl Math Comput 169:897-904. doi:10.1016/j.amc.2004.09.092

Jahanshahloo GR, Hosseinzadeh Lotfi F, Shoja N, Tohidi G, Razavyan S (2005b) A one-model approach to classification and sensitivity analysis in DEA. Appl Math Comput 169:887-896. doi:10.1016/j.amc.2004.09.068

Jahanshahloo GR, Hosseinzadeh Lotfi F, Zohrehbandian M (2005c) Finding the piecewise linear frontier production function in data envelopment analysis. Appl Math Comput 163:483-488. doi:10. 1016/j.amc.2004.02.016

Jahanshahloo GR, Hosseinzadeh Lotfi F, Zhiani Rezai H, Rezai Balf F (2007) Finding strong defining hyperplanes of production possibility set. Eur J Oper Res 177:42-54. doi:10.1016/j.ejor. 2005.11.031
Jahanshahloo GR, Shirzadi A, Mirdehghan SM (2009) Finding strong defining hyperplanes of PPS using multiplier form. Eur J Oper Res 194:933-938. doi:10.1016/j.ejor.2008.01.053

Lachhwani K, Poonia MP (2012) Mathematical solution of multilevel fractional programming problem with fuzzy goal programming approach. J Ind Eng Int 8(16):1-11. doi:10.1186/2251-712x-8-16

Pitchipoo P, Venkumar P, Rajakarunakaran S (2013) Modeling and development of a decision support system for supplier selection in the process industry. J Ind Eng Int 9(23):1-15. doi:10.1186/ 2251-712x-9-23

Seiford LM, Zhu J (1998) Stability regions for maintaining efficiency in data envelopment analysis. Eur J Oper Res 108:127-139. doi:10.1016/S0377-2217(97)00103-3

Seiford LM, Zhu J (1999) Infeasibility of super-efficiency data envelopment analysis models. INFOR 37(2):174-187

Singh CJ, Jain M, Kumar B (2012) Analysis of M/G/1 queueing model with state dependent arrival and vacation. J Ind Eng Int 8(2):1-8. doi:10.1186/2251-712x-8-2

Tabrizi BH, Razmi J (2013) A multi-period distribution network design model under demand uncertainty. J Ind Eng Int 9(13):1-9. doi:10.1186/2251-712x-9-13

Zhu J (1996) Robustness of the efficient DMUs in data envelopment analysis. Eur J Oper Res 90:451-460. doi:10.1016/03772217(95)00054-2

Zhu J (2001) Super-efficiency and DEA sensitivity analysis. Eur J Oper Res 129:443-455. doi:10.1016/S0377-2217(99)00433-6 